\title{
Croissance et décroissance des réseaux, approches méthodologiques
}

\author{
Françoise Bahoken* et Serge Lhomme** \\ Septembre 2016 \\ *IFSTTAR et UMR GÉographie-Cités \\ **Lab'Urba - Créteil Paris Est \\ groupe f.m.r.
}

Ce dossier est issu de la troisième journée d'études du groupe fmr (flux, matrice, réseaux), qui s'est tenue le 25 septembre 2014 à l'Institut des Systèmes Complexes (ISC), à Paris. Cette journée intitulée Croissance et décroissance des réseaux. Approches méthodologiques, avait pour objectif d'explorer les méthodes d'analyse de la dynamique des réseaux spatiaux dans le temps. Le recueil des communications présentées est disponible en ligne (Beauguitte, 2014).

Une sélection de quatre communications a finalement été retenue dans le cadre de ce dossier. Olivier Bonin analyse la construction de la couche d'information géographique symbolisant un réseau routier cartographié à différentes époques. L'auteur met ainsi l'accent sur la sémantique du réseau et la modélisation cartographique qui en découlent. Angélique Palle s'intéresse au réseau d'électricité européen : elle interroge sa dynamique couplée à des questionnements de nature prospective. Actuellement, ces réseaux sont paradoxalement confrontés à une double logique : une logique de croissance, visant à les renforcer (densification d'un réseau européen vulnérable) et une autre de décroissance, du fait de l'avènement de nouvelles technologies (énergies renouvelables, productions d'électricité locales...). Claire Lagesse, Philippe Bonnin, Patricia Bordin et Stéphane Douady introduisent une nouvelle méthode de caractérisation des propriétés topologiques et géométriques des réseaux viaires spatialisés, reposant sur la notion de 《voie ». L'approche, focalisant l'attention sur la construction d'indicateurs, révèle une hiérarchie des voies dont la morphogénèse conduit les auteurs à retracer leur historique. Julie Prud'homme mobilise, quant à elle, des réseaux spatiaux déjà constitués pour en déduire leur contribution à l'estimation de 
polluants liés au trafic routier dont ils font l'objet.

La présentation de ces travaux focalise d'abord l'attention sur les aspects formels de l'objet réseau, tel qu'il est appréhendé par les recherches étudiant un réseau viaire (Bonin; Prudhomme; Lagesse et alii). En effet, bien que ces travaux concernent le même objet, les termes utilisés pour le décrire diffèrent, les inscrivant alors dans un cadre théorique donné. C'est pourquoi, de ce point de vue, il n'est pas surprenant que les analyses s'appuient sur des approches très différentes. Successivement descriptives, modélisatrices ou discursives, toutes soulignent les difficultés méthodologiques d'une analyse historique ou dynamique des réseaux.

\section{Terminologies du réseau viaire}

Trois recherches s'intéressent au réseau viaire, en mobilisant plusieurs expressions : le « réseau viaire de transport », le « réseau planaire », le « réseau quasi-planaire », le 《réseau non planaire », la 《 route » ou le 《réseau », valué ou non. Lagesse et alii évoquent même un « objet complexe continu » qui correspond formellement à un ensemble de chaînons. Si le thème de la journée n'était pas de discuter de la définition de l'objet réseau, la variété des qualificatifs utilisés correspond, de notre point de vue, à un résultat important. Ces différences terminologiques ne sont pas de simples écarts de langages, ils matérialisent l'inscription des auteurs dans un cadre théorique donné.

En effet, derrière cette variété de termes, les articles se distinguent selon qu'ils ont recours ou non à la notion de graphe spatialisé, c'est-à-dire selon qu'ils utilisent ou non une forme d'abstraction et de représentation géométrique d'un réseau dans un espace géographique. Les travaux ayant recours à la notion de graphe se placent alors dans une perspective modélisatrice; les auteurs mettent notamment en œuvre des indicateurs pour décrire la structure et les caractéristiques de ce réseau, à l'inverse des approches plus descriptives qui mobilisent des méthodes qualitatives.

Plus précisément, il est intéressant de montrer la diversité des termes utilisés et en particulier des notions clés. Tandis que Bonin s'interroge sur la définition de la route - sa notion clé - et qu'il ne mentionne ni la voie ni l'arc, mais évoque le tronçon en bibliographie, Lagesse et alii questionnent celle de voie; Prudhomme, le tronçon. Le tableau 1 positionne les trois textes au regard de ces quatre notions, dans un degré d'abstraction formelle croissant depuis la route à son expression géométrique la plus simplifiée, sous la forme d'un arc.

Les travaux de Lagesse et alii introduisent la notion de «voie », ce qui leur permet de dépasser la formalisation classique du réseau sous la forme de nœuds et d'arcs, en faisant évoluer ces objets. Les auteurs prennent ainsi en compte une caractéristique géométrique des liens, l'orientation. C'est pour- 
Figure 1 - Notions clés de description d'un réseau de transport routier, selon différents auteurs

\begin{tabular}{|c|c|c|c|c|}
\hline Auteur/ notion & Route & Voie & Tronçon & Arc \\
\hline O. Bonin & Réseau de routes* & & $\begin{array}{l}\text { Mentionné en } \\
\text { bibliographie }\end{array}$ & \\
\hline J. Prud'homme & $\begin{array}{l}\text { Le réseau routier } \\
\text { est utilisé en } \\
\text { complément du } \\
\text { tronçon de route. }\end{array}$ & $\begin{array}{l}\text { L'aspect littéral du } \\
\text { terme de voie est } \\
\text { mobilisé pour } \\
\text { préciser un } \\
\text { contexte (ex. une } \\
\text { voie de montagne). }\end{array}$ & $\begin{array}{l}\text { Le tronçon de } \\
\text { route correspond } \\
\text { formellement au } \\
\text { réseau routier. }\end{array}$ & $\begin{array}{l}\text { Une citation de } \\
\text { l'arc au début du } \\
\text { texte est proposée } \\
\text { pour définir le } \\
\text { graphe. }\end{array}$ \\
\hline $\begin{array}{l}\text { C. Lagesse } \\
\text { et alii }\end{array}$ & $\begin{array}{l}\text { Une citation du } \\
\text { terme de route est } \\
\text { proposée en } \\
\text { complément de la } \\
\text { voie. }\end{array}$ & $\begin{array}{l}\text { La voie, issue de } \\
\text { l'arc, est utilisée } \\
\text { pour formaliser un } \\
\text { objet, dans le cadre } \\
\text { de la théorie des } \\
\text { graphes. }\end{array}$ & $\begin{array}{l}\text { Le tronçon est cité } \\
\text { deux fois, en } \\
\text { référence à la } \\
\text { syntaxe spatiale. }\end{array}$ & $\begin{array}{l}\text { L'arc est très } \\
\text { présent dans le } \\
\text { texte (voir } \\
\text { bibliographie), il } \\
\text { permet } \\
\text { l'émergence de la } \\
\text { voie. }\end{array}$ \\
\hline
\end{tabular}

quoi ils s'inscrivent dans le champ de la syntaxe spatiale et de la théorie des graphes. Le réseau y est appréhendé dans son abstraction formelle la plus mathématique.

Prud'homme, qui utilise le terme de «tronçon», appréhende le réseau en tant que «support» de déplacement des objets mobiles, inscrivant alors pleinement son approche dans le champ de la géographie des transports. L'analyse est mobilisée dans le cadre d'une approche globale, résolument territoriale et opérationnelle, proche des thématiques d'aménagement. Cela explique le recours de l'auteure à une approche formelle, dans une perspective d'enrichissement thématique qui porte, en l'occurrence, sur la modélisation des polluants atmosphériques liés au trafic routier.

Bonin étudie, de son côté, le « réseau de routes», sans recourir à la modélisation sous la forme de « graphe» qui en découle, considérant cette abstraction comme une forme trop simplificatrice de cet objet complexe. Ainsi, pour construire le réseau, l'auteur propose de le définir précisément. Il montre, par exemple, que, dans le cadre d'une reconstruction du processus historique de cartographie du réseau, apparaît la difficulté d'une analyse historique fondée sur l'examen d'un simple tracé de route résultant de nombreux choix de modélisation graphique. Sans surprise, il n'y a pas de construction d'indicateurs sur le réseau chez cet auteur, car il n'en est pas à ce stade réflexif : son analyse portant davantage sur la construction de l'objet route et non sur son exploitation.

Ces différentes postures théoriques et méthodologiques se retrouvent également dans la manière dont les auteurs appréhendent l'aspect dynamique 
du réseau.

\section{Modélisation de la dynamique du réseau}

Dans les faits, la question de la dynamique du réseau est peu présente dans les différents textes reçus, même si cette question fut débattue lors des présentations orales et des discussions qui s'en suivirent. Prud'homme aborde cette question par l'analyse des déplacements automobiles sur ce réseau, c'est-à-dire exclusivement en termes de «flux »; l'auteure ne questionne pas son évolution structurelle. Bonin l'évoque, dans sa volonté de questionner la dynamique intrinsèque des graphes, en posant la question de la pertinence de l'objet : qu'est ce qu'une route?; de sa construction sur la carte : comment est représentée une route?; et de sa permanence dans le temps : que reste-t-il de cette route? In fine, seule Palle a réellement mobilisé la question de la dynamique d'un réseau. Son approche, caractérisée par une démarche qualitative fondée sur l'analyse d'entretiens d'experts, traite également une question encore plus complexe : celle de la prospective. À l'inverse, la question de la dynamique fut presque inexistante dans les travaux relevant d'approches davantage modélisatrices.

Cette lacune ayant notamment été soulevée par les auditeurs, elle a pu faire l'objet d'un débat plus général lors de la séance de questions avec la salle. Deux grandes familles de verrous ont ainsi pu être identifiées, selon qu'elles s'intéressent aux aspects d'ordre méthodologique ou au niveau sémantique de la caractérisation du réseau.

Les verrous méthodologiques sont liés aux possibilités d'analyse de différents jeux de données portant sur des objets similaires, considérés à différentes dates. Ils concernent, par exemple, i) les problèmes d'appariement pour détecter les changements survenus sur un réseau au cours du temps. Lagesse et alii évoquent d'ailleurs la difficulté de reconnaissance d'une même voie à différentes époques. Les verrous méthodologiques sont également liés ii) à des erreurs de saisie ou de contrôle de l'émergence d'un élément du réseau ainsi qu'à iii) la disponibilité de données similaires observées à différentes dates.

Les verrous sémantiques portent sur la signification de l'objet qui a pu évoluer au cours du temps. S’il est théoriquement possible de comparer des réseaux dans le temps, ces réseaux ne revêtent pas toujours la même signification. C'est pourquoi Bonin insiste sur la sémantique et la nécessité de définir l'objet route à différentes époques.

De nouvelles approches d'analyses de l'objet réseau ont ainsi émergé. Elles sont, par exemple, fondées sur la syntaxe spatiale souvent mobilisée dans le champ des transports. Cette évolution résulte d'une complexification de l'objet réseau qui s'explique notamment par sa densification (l'augmentation du nombre de voies au cours du temps). Le réseau viaire est désormais 
plus complet, ce qui n'était pas le cas dans le passé, avant le XVIIe siècle. Aujourd'hui, un réseau de transport se conçoit de manière unifiée, c'est-à-dire comme une composante connexe unique, ce qui n'était pas nécessairement le cas auparavant. En outre, la fragmentation de ce réseau révèle alors sa vulnérabilité. Cette acception de la complexification, présente au niveau national - dans l'exemple donné par Palle du réseau d'électricité -, s'observe aujourd'hui à l'échelle européenne.

Ce point questionne par ailleurs la permanence de la signification du réseau dans le temps et, par conséquent, la possibilité de lui appliquer des indicateurs similaires à différents moments. La comparaison de la densité d'un réseau supposé similaire aux XVIIe et XXIe siècles est-elle vraiment pertinente? Une hypothèse qui mériterait d'être vérifiée.

La complexification du réseau actuel s'accompagne de l'émergence de nouveaux concepts qu'il est probablement vain de vouloir étudier de manière dynamique, du moins dans un premier temps. La notion d'arc a-t-elle un sens pour des voies non carrossables, en comparaison de voies pavées? Ou bien prend-elle des modalités différentes en fonction du temps?

C'est pourquoi l'absence d'approches modélisatrices de la dynamique des réseaux dans les textes composant ce dossier s'explique, probablement, par le fait que ces réseaux - ce que Bonin décrit bien pour les réseaux viaires - sont par définition instables dans le temps. Leur nature d'ensemble est intrinsèquement évolutive. Palle semble, en effet, montrer que la question de la croissance et de la décroissance du réseau d'électricité est liée aux processus de transformation à l'œuvre, apparition des smart grids et de «[...] sources de production locale d'énergies renouvelables ».

Pour finir, il existe sans doute toujours une fenêtre temporelle par laquelle des possibilités d'analyse de la dynamique des réseaux - croissance, décroissance, densification... - sont envisageables. Néanmoins, l'une des questions essentielles nous semble celle de savoir si, en l'absence d'un changement profond de la nature d'un réseau, il peut y avoir un changement structurel radical.

\section{Bibliographie}

Beauguitte L. (coord.), 2014, Croissance et décroissance des réseaux, Actes de la troisième journée d'étude du groupe fmr (flux, matrices, réseaux), [en ligne] URL : http://halshs.archives-ouvertes.fr/ (consulté le 4 juillet 2016)

Bonin O., 2016, Analyse de la croissance de réseaux de transport sur le moyen terme à partir de sources cartographiques : comment utiliser la carte de Cassini comme source de données?, Flux 2016/3 (105).

Lagesse C., Bonnin P., Bordin P., Douady S., 2016, Méthodologie de modélisation et de caractérisation des réseaux spatiaux. Application au réseau 
viaire de Paris, Flux 2016/3 (105).

Palle A., 2016, Les dynamiques d'évolution des réseaux de transport d'électricité européens, des réseaux techniques entre croissance et décroissance, Flux 2016/3 (105).

Prud'homme J., 2016, Le réseau routier, déterminant structurel de la quantité de polluants émis par les déplacements automobiles, Flux 2016/3 (105). 